\title{
Condom Versus Indwelling Urinary Catheters: A Randomized Trial
}

\author{
Sanjay Saint, MD, MPH, ${ }^{* \dagger}$ Samuel R. Kaufman, MA, ${ }^{\dagger \neq}$ Mary A. M. Rogers, PhD, ${ }^{\dagger \neq}$ \\ Paul D. Baker, ARNP, ${ }^{\mathcal{S}}$ Kathleen Ossenkop, ARNP, ${ }^{\mathcal{S}}$ and Benjamin A. Lipsky, MD ${ }^{\mathcal{S}}$
}

OBJECTIVES: To compare condom and indwelling urinary catheters in terms of infection risk and patient satisfaction.

DESIGN: A prospective, randomized, unblinded, controlled trial.

SETTING: An academically affiliated Veterans Affairs Medical Center.

PARTICIPANTS: Hospitalized men aged 40 and older who required a urinary collection device.

MEASUREMENTS: The incidence of adverse outcomes (bacteriuria, symptomatic urinary tract infection (UTI), or death) and patient device-related satisfaction as determined according to a questionnaire. Dementia status was recorded to assess effect modification by the presence of dementia.

RESULTS: Seventy-five subjects were randomized: 41 receiving an indwelling catheter and 34 a condom catheter. The incidence of an adverse outcome was 131/1,000 patient-days with an indwelling catheter and 70/1,000 patient-days with a condom catheter $(P=.07)$. The median time to an adverse event was 7 days in the indwelling group and 11 days in the condom group. After adjusting for other risk factors, it was found that condom catheter use reduced adverse outcomes $(P=.04)$. Patients without dementia who had an indwelling catheter were approximately five times as likely to develop bacteriuria or symptomatic UTI or to die (hazard ratio $=4.84,95 \%$ confidence interval $=$ 1.46-16.02) as those with a condom catheter $(P=.01)$. Patients reported that condom catheters were more comfortable $(P=.02)$ and less painful $(P=.02)$ than indwelling catheters.

From the *Center for Practice Management and Outcomes Research, Ann Arbor Department of Veterans Affairs Health Services Research and Development Center of Excellence, Ann Arbor, Michigan; ${ }^{\dagger}$ Department of Internal Medicine, Division of General Medicine, University of Michigan, Ann Arbor, Michigan; ${ }^{\ddagger}$ Department of Veterans Affairs/University of Michigan Patient

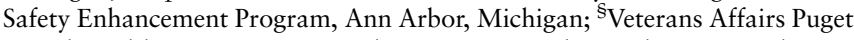
Sound Health Care System, Seattle Division, Seattle, Washington; and

"Department of Medicine, School of Medicine, University of Washington, Seattle, Washington.

Address correspondence to Sanjay Saint, MD, MPH, Room 7E08, 300 NIB_Campus Box 0429, Ann Arbor, MI 48109. E-mail: saint@umich.edu DOI: $10.1111 / \mathrm{j} .1532-5415.2006 .00785 . \mathrm{x}$
CONCLUSION: The use of condom catheters is less likely to lead to bacteriuria, symptomatic UTI, or death than the use of indwelling catheters. This protection is especially apparent in men without dementia. J Am Geriatr Soc 54:1055-1061, 2006.

Key words: urinary catheter; urinary tract infection; bacteriuria; cognitive impairment

$M$ illions of Americans undergo urinary bladder catheterization annually in various healthcare settings. ${ }^{1}$ Up to $25 \%$ of hospitalized patients have a urinary catheter placed during their stay, 2,3 and $5 \%$ to $15 \%$ of nursing home residents have an indwelling catheter. ${ }^{4-7}$ These urinary catheters often cause considerable discomfort and embarrassment to patients. ${ }^{8-10}$ They are also the proximate cause of the vast majority of healthcare-associated urinary tract infections (UTIs), ${ }^{11,12}$ which account for more than onethird of nosocomial infections in acute care hospitals. ${ }^{3,13-15}$ Catheter-related UTI is also the most common infection in long-term care facilities and is the likely source of twothirds of febrile episodes in this setting. ${ }^{16}$ The substantial morbidity associated with urinary catheter-related infection also generates additional healthcare costs. ${ }^{10,17,18}$

Although the health risks of indwelling urinary catheterization are well known, the risks and benefits of alternatives have not been adequately studied. For male patients who do not retain urine, external urine collection systems are an option, but the conflicting results of the few available studies have left their role in hospitalized or long-term care patients unclear. ${ }^{19-22}$ A recent systematic review found that no conclusions could be drawn from existing randomized or quasi-randomized controlled trials regarding when to use various types of catheters in managing patients with a neurogenic bladder. ${ }^{23}$ In light of how frequently these two types of urinary collection devices are used and the uncertainty of their associated infection risks, several authorities have called for a comparative trial.7,24

A randomized trial comparing external (condom) catheters with indwelling urethral catheters in hospitalized men who required a urinary collection device was therefore 
conducted. It was hypothesized that substituting a condom catheter for an indwelling one might decrease the incidence of bacteriuria and symptomatic UTI, but because a patient can more easily manually manipulate a condom catheter than an indwelling one, the hypothesis that a condom catheter might be less beneficial in cognitively impaired patients was also tested. ${ }^{25}$ Recognizing the importance of patient preferences, patient satisfaction with the two types of urinary collection devices was also compared.

\section{METHODS}

\section{Setting}

All enrolled patients were hospitalized at the Veterans Affairs Puget Sound Health Care System (VAPSHCS) in Seattle, Washington, from 1997 through 2001. This University of Washington-affiliated primary care and referral center has full medical and surgical services, three intensive care units, a spinal cord injury unit, a bone marrow transplant unit, and a nursing home care unit. The Human Subjects Review Board at the University of Washington and the Research and Development Committee at VAPSHCS approved this research project. All enrolled patients, or for those with cognitive impairment, their next of kin, provided informed consent.

\section{Study Subjects}

Potential subjects were male veterans aged 40 and older who were newly admitted to or currently hospitalized in the nursing home care unit or an acute medicine, neurology, or rehabilitation ward within the VAPSHCS from August 1997 through March 2001. A study nurse screened patients in these units who required a urinary collection device for possible enrollment. For patients already hospitalized, the study nurse reviewed the medical record, including nursing notes and physician orders, to find those who required a urinary collection device. For a newly admitted patient, the study nurse contacted the resident or attending physician to inquire whether the patient needed a urinary collection device for urinary incontinence or for monitoring urinary output.

Patients were excluded if they had evidence, according to history or previous diagnostic studies, of urinary retention or severe bladder outlet obstruction; had any congenital or acquired urogenital abnormality that might preclude using a condom or an indwelling catheter (e.g., urethral stricture, recent urological surgery or pelvic radiation, hypospadias); were bacteriuric; required frequent monitoring of urinary output (e.g., due to acute renal failure or congestive heart failure); were unlikely to live for at least 2 weeks; were receiving systemic antibiotic therapy for any reason; were scheduled to be discharged within a few days; were unable to provide informed consent and had no appropriate surrogate; had azotemia; or had a physician who recommended against enrolling them.

\section{Intervention}

Each enrolled patient was randomized to receive an indwelling urethral catheter (standard, balloon-tipped, silicone-coated latex Foley) or a condom catheter (silicone, latex-free, self-adhering male external). Mentor Corpora- tion (Santa Barbara, CA) provided the condom catheter used (Clear Advantage), a transparent one-piece unit that comes in five sizes and is connected to a 2,000-mL drainage bag.

\section{Outcome Measures}

The main outcome measure was the incidence of developing bacteriuria (defined as $\geq 10^{3}$ colony-forming units per $\mathrm{mL}$ of a single or predominant species of bacteria). ${ }^{26}$ The study nurse obtained a sample of urine for culture at the time of randomization and requested that another be obtained daily for the duration of the subject's enrollment in the study. For those with an indwelling catheter, the specimen of urine was collected using aseptic needle puncture from the sampling port in the drainage tubing. ${ }^{27}$ For those with a condom catheter, the first voided sample within an hour after each daily catheter change was obtained from the sampling port in the drainage tubing. ${ }^{28,29}$ The urine specimens were sent to the VAPSHCS microbiology laboratory and processed with the usual microbiological techniques. Study staff examined the laboratory results and noted the date and time at which a sample satisfied the study definition of bacteriuria. The maximum duration of follow-up for each enrolled patient was 30 days. Two additional clinical outcomes were also monitored: patient mortality during the study period and the development of a symptomatic UTI (defined as bacteriuria accompanied by onset of one or more of the following symptoms or signs: fever $>38^{\circ} \mathrm{C}$; dysuria or other irritative voiding symptoms; or suprapubic, flank, or pelvic pain thought to be related to the urinary tract). Finally, using a previously developed standardized questionnaire, ${ }^{9}$ the patient's satisfaction with the urinary device he was wearing was assessed. The questionnaire contained items quantifying discomfort, restriction in movement, pain, embarrassment, and inconvenience.

\section{Randomization and Blinding}

Patients were randomized using concealed allocation. The group to which a patient was assigned (condom catheter or indwelling catheter) was determined using a random number table and block randomization. Group assignments were designated in sequence, using sealed opaque envelopes that were concealed to patients, data collectors, and staff at the study site until the study nurse assigned the intervention. Subject assignment and study hypotheses were concealed from the laboratory technicians who processed and interpreted the urine cultures (the primary outcome).

\section{Data Collection}

For each enrolled patient, the following clinical information was obtained: age; primary medical condition; history of diabetes mellitus, malignancy (other than basal cell or squamous cell skin cancer), prostate disorders or prostate surgery, previous urethral catheterization, infection with human immunodeficiency virus, or UTI; treatment with any systemic corticosteroid in the previous week or treatment with any chemotherapeutic agent in the previous 2 weeks; most recent serum creatinine value; and circumcision status. The study nurse assessed the patient's mental status using an index consisting of the first eight items of the standard Mini-Mental State Examination (abbreviated 
Mini-Mental State Examination (aMMSE)). The following clinical information was obtained daily: results of urine cultures, symptoms or signs of UTI (defined above), presence of fever, and any adverse events associated with the catheter (e.g., gross hematuria, genital ulceration, erosion, or excoriation).

\section{Data Analysis}

Statistical analyses were based on the intention-to-treat principle. The Pearson chi-square test or Fisher exact test (when expected cell counts were less than 5) was used for baseline comparisons of categorical data, the Student $t$ test for differences in means, and the Mann-Whitney $U$ test for differences in medians. Incidence rates (events per 1,000 patient-days) for the outcomes were calculated with $95 \%$ confidence intervals, as were the median times (days) to a given outcome. Survival analysis was used to assess the efficacy of the intervention on the incidence of bacteriuria and secondary endpoints. The Kaplan-Meier product limit estimates were graphed, and differences in curves were evaluated using the log-rank test. To examine risk factors contributing to the development of bacteriuria and the secondary outcomes in each group while adjusting for potential confounders, Cox proportional hazards regression was used. Effect modification by dementia was considered, because a priori evidence suggested differing effects based on this variable. Specifically, it was anticipated that any potential beneficial effect of the condom catheter would be more evident in patients without dementia, because they are less likely to manipulate their catheters manually. ${ }^{25}$ To ensure that all randomized subjects were included in the regression models, missing information on covariates was imputed. Missing values for prior catheterization (3 cases), previous UTI ( 4 cases), and aMMSE ( 3 cases) were replaced with median values for the patient's group (condom or indwelling). The two patients whose dementia status was missing but who scored 0 on the aMMSE were classified as having dementia. Hazard regression models were estimated with and without the imputed values, and no appreciable difference in results was found.

Pearson chi-square tests or Fisher exact tests were used for analyses of the indices of patient satisfaction by type of catheter. For all analyses, alpha was set at .05, two-sided.

\section{RESULTS}

During the 3.5 years of the study, 4,241 patients were screened for eligibility; 4,144 did not qualify based on the inclusion and exclusion criteria, and 22 refused to participate. Of the 75 eligible consenting patients, 34 were randomized to the condom catheter group and 41 to the indwelling catheter group. The overall median length of hospital stay after enrollment was 3 days ( 2 days in the indwelling catheter group, 4 days in the condom catheter group, $P=.11$ for the difference). During the course of the trial, daily urine collection was terminated for various medical reasons for four patients, and seven patients withdrew consent (4 in the condom group, 3 in the indwelling group). Events for these patients were treated as censored at the time of the termination or withdrawal.

Baseline characteristics of the subjects are shown in Table 1. The mean age of the subjects in the two groups was similar (73.4 and 73.7), as was the percentage with diabetes mellitus $(35.3 \%$ and $25.6 \%, P=.37)$ and malignancy $(17.6 \%$ and $18.4 \%)$. Almost one-quarter had a history of prostatitis or prostate surgery, and the majority had undergone a prior urethral catheterization. By chance, a significantly $(P<.01)$ greater percentage of patients with dementia were assigned to the condom catheter group $(60.6 \%)$ than to the indwelling group $(20.5 \%)$. Not surprisingly, therefore, mean aMMSE scores were lower in the condom catheter group.

Table 1. Comparison of Subjects Assigned to Condom Catheter and Indwelling Catheter Groups at Baseline

\begin{tabular}{|c|c|c|c|}
\hline Characteristic & $\begin{array}{l}\text { Condom } \\
\text { Catheter }\end{array}$ & $\begin{array}{l}\text { Indwelling } \\
\text { Catheter }\end{array}$ & $P$-value* \\
\hline Age, mean $\pm \mathrm{SD}$ & $73.4 \pm 11.0$ & $73.7 \pm 10.0$ & .92 \\
\hline \multicolumn{4}{|l|}{ Comorbidity, n (\%) } \\
\hline Diabetes mellitus & $12(35.3)$ & $10(25.6)$ & .37 \\
\hline Current malignancy & $6(17.6)$ & $7(18.4)$ & .93 \\
\hline Dementia, n (\%) & $20(60.6)$ & $8(20.5)$ & $<.01$ \\
\hline Abbreviated Mini-Mental State Examination, mean \pm SD & $16.3 \pm 7.9$ & $20.7 \pm 6.9$ & .01 \\
\hline \multicolumn{4}{|l|}{ Medications of note, $\mathrm{n}(\%)$} \\
\hline Corticosteroid use in last 7 days & $6(18.8)$ & $8(22.2)$ & .72 \\
\hline Antibiotic use last 72 hours & $18(52.9)$ & $19(50.0)$ & .80 \\
\hline \multicolumn{4}{|l|}{ Genitourinary issues, $\mathrm{n}(\%)$} \\
\hline Circumcised & $34(100.0)$ & $35(92.1)$ & .24 \\
\hline Prior urethral catheterization & $26(76.5)$ & $32(84.2)$ & .41 \\
\hline Prior urinary tract infection & $10(30.3)$ & $11(28.9)$ & .90 \\
\hline History of prostatitis & $2(6.3)$ & $2(5.4)$ & 1.00 \\
\hline History of prostate surgery & 7 (20.6) & 9 (23.1) & .80 \\
\hline
\end{tabular}

* Pearson chi-square test for categorical data with expected counts $\geq 5$; Fisher exact test for categorical data with expected counts $<5$; Student $t$ test for differences of means (equal variances).

$\mathrm{SD}=$ standard deviation . 
Table 2. Comparison of Incidence of, and Median Time to, Outcomes of Interest in Subjects Randomized to Condom Catheter or Indwelling Catheter

\begin{tabular}{|c|c|c|c|}
\hline Outcome & $\begin{array}{l}\text { Condom Catheter } \\
\qquad(n=34)\end{array}$ & $\begin{array}{l}\text { Indwelling Catheter } \\
\qquad(\mathrm{n}=41)\end{array}$ & $P$-value* \\
\hline \multicolumn{4}{|l|}{ Bacteriuria $^{\dagger}$} \\
\hline Time to outcome, days, median (SE) & $13(0.9)$ & $7(0.4)$ & .15 \\
\hline Incidence per 1,000 patient-days $(95 \% \mathrm{Cl})$ & $61(35-104)$ & $111(69-178)$ & .11 \\
\hline \multicolumn{4}{|l|}{ Bacteriuria, symptomatic UTI, or death } \\
\hline Time to outcome, days, median (SE) & $11(1.0)$ & $7(0.7)$ & .09 \\
\hline Incidence per 1,000 patient-days $(95 \% \mathrm{Cl})$ & $70(42-116)$ & $131(85-203)$ & .07 \\
\hline
\end{tabular}

During the study period, 30 patients developed bacteriuria: $13(38.2 \%)$ in the condom catheter group and 17 $(41.5 \%)$ in the indwelling catheter group (Table 2). Of the six patients who died during the trial, two $(5.9 \%)$ were in the condom catheter group, and four $(9.8 \%)$ were in the indwelling catheter group. The incidence of bacteriuria in patients with a condom catheter was 61/1,000 patient-days, compared with $111 / 1,000$ patient-days in those with an indwelling catheter $(P=.11)$. In those who developed bacteriuria, the median time to onset was shorter in patients with an indwelling catheter ( 7 days) than in those with a condom catheter (13 days) $(P=.15)$.

Of those in the condom catheter group, $15(44.1 \%)$ experienced one or more adverse events (i.e., bacteriuria, symptomatic UTI, or death) during the trial, compared with $20(48.8 \%)$ in the indwelling catheter group. The incidence of an adverse event was 70/1,000 patient-days in those with a condom catheter and 131/1,000 patient-days in those with an indwelling catheter $(P=.07)$. The median time to an adverse event was 11 days in the condom catheter group and 7 days in the indwelling catheter group $(P=.09)$.

Unadjusted Kaplan-Meier curves comparing patients in the condom catheter group with those in the indwelling catheter group showed no statistically significant difference in the incidence of an adverse event for patients with dementia. However, they did show a significant difference when the analysis was restricted to those without dementia, where the incidence was lower in the condom catheter patients $(P=.04)$.

Table 3 shows the hazard ratios (HRs) for incurring one or more of the adverse events. The unadjusted HR was 1.82 (95\% confidence interval $(\mathrm{CI})=0.90-3.67)$ for all patients and $3.47(95 \% \mathrm{CI}=0.94-12.74)$ for patients without dementia. After adjustment for age, aMMSE score, prior UTI, and prior urethral catheterization, the incidence of an adverse study outcome was statistically significantly lower in the condom catheter group than in the indwelling catheter group. The risk of bacteriuria, symptomatic UTI, or death was twice as high in patients with an indwelling catheter. This was evident whether the baseline hazard function differed by dementia status $(\mathrm{HR}=2.26,95 \% \mathrm{CI}=1.10-4.62)$ or was the same across all patients $(\mathrm{HR}=2.11,95 \%$ $\mathrm{CI}=1.03-4.31$ ). When the analysis was restricted to patients without dementia, the effect was stronger, with an HR of $4.84(95 \% \mathrm{CI}=1.46-16.02)$; this indicates that the risk of bacteriuria, symptomatic UTI, or death was five

Table 3. Hazard Ratios for Bacteriuria, Symptomatic Urinary Tract Infection (UTI), or Death Comparing Patients Randomized to an Indwelling Catheter with Those Randomized to a Condom Catheter (Reference)

\begin{tabular}{|c|c|c|c|c|}
\hline Regression Model & $\mathrm{n}$ & Hazard Ratio & 95\% Confidence Interval & $P$-value \\
\hline \multicolumn{5}{|l|}{ Unadjusted } \\
\hline All patients & 75 & 1.82 & $0.90-3.67$ & .09 \\
\hline Patients without dementia & 44 & 3.47 & $0.94-12.74$ & .06 \\
\hline Patient with dementia & 31 & .86 & $0.23-3.27$ & .83 \\
\hline \multicolumn{5}{|l|}{ Adjusted* } \\
\hline All patients & 75 & 2.11 & $1.03-4.31$ & .04 \\
\hline All patients, stratified by dementia ${ }^{\dagger}$ & 75 & 2.26 & $1.10-4.62$ & .03 \\
\hline Patients without dementia & 44 & 4.84 & $1.46-16.02$ & .01 \\
\hline Patients with dementia & 31 & 1.20 & $0.33-4.35$ & .78 \\
\hline
\end{tabular}

* Adjusted for age, abbreviated Mini-Mental State Examination score, history of UTI, and history of catheterization.

${ }^{\dagger}$ Baseline hazard functions differed by dementia status (yes/no). 

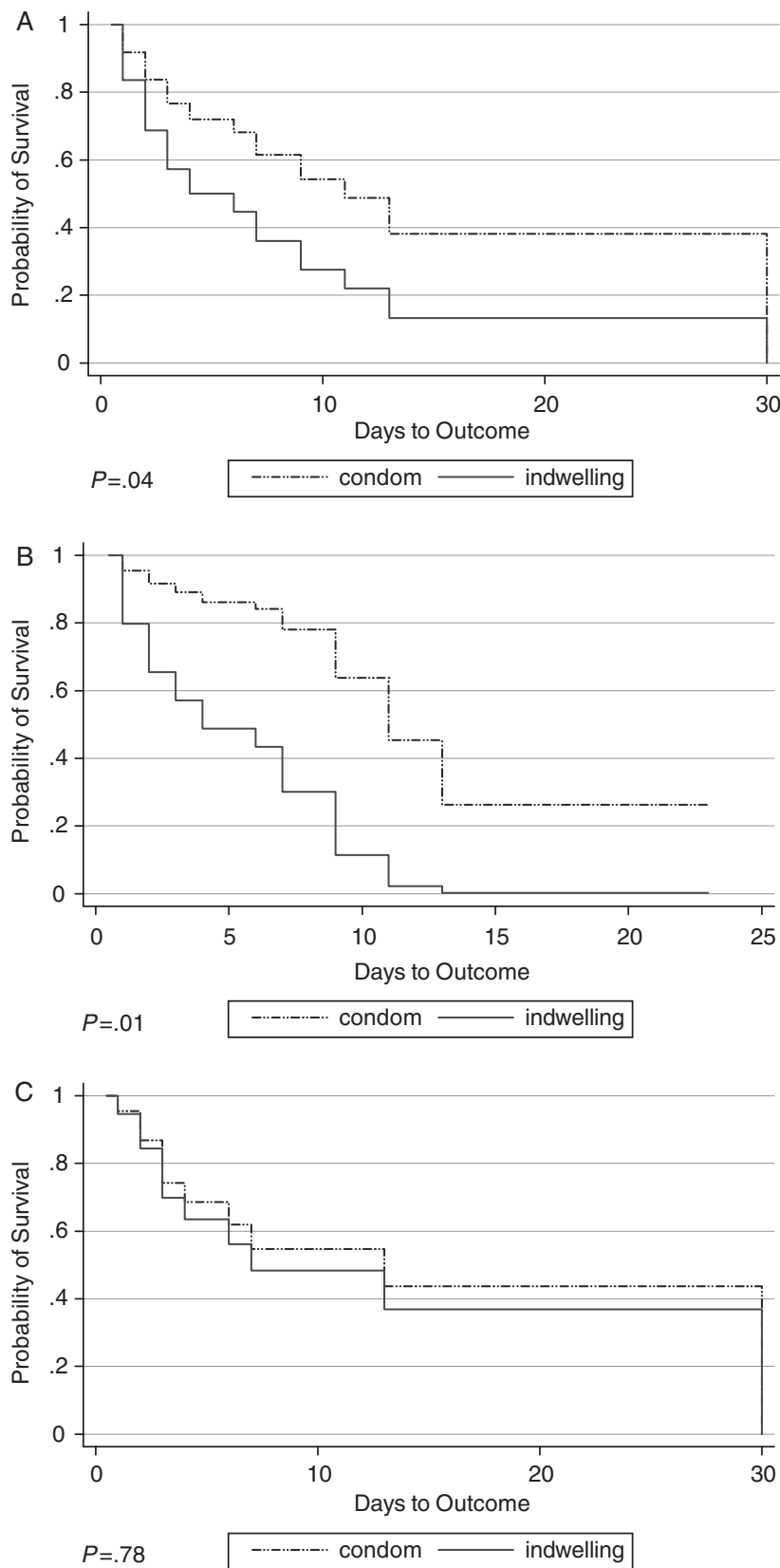

Figure 1. Adjusted* survival curves for a study-related adverse outcome: ${ }^{\dagger}$ A. All patients; B. Patients without dementia; C. Patients with dementia. *Adjusted for age, abbreviated Mini-Mental State Examination score, history of urinary tract infection, and history of catheterization. ${ }^{\dagger}$ Bacteriuria, symptomatic urinary tract infection, or death.

times greater in patients without dementia who had an indwelling catheter. One outcome in particular-bacteriuria - which was by far the most frequent, greatly affected these results. The results would not have been substantially different if bacteriuria alone had been considered. In Figure 1A, the survival curves generated from the adjusted Cox proportional hazards regression model for all patients show a significantly worse outcome for patients in the indwelling catheter group $(P=.04)$. Figures $1 \mathrm{~B}$ and $\mathrm{C}$ show that the difference in adverse outcomes was even greater for patients without dementia $(P=.01)$ but was not significantly different for those with dementia $(P=.78)$.

Based on questionnaire results, patients with a condom catheter were more likely to report their device to be comfortable $(P=.02)$ and not painful $(P=.02)$ than were patients with an indwelling catheter (Table 4). On other measures of preference, there were no significant differences.

\section{DISCUSSION}

Urinary catheterization is a necessity for a substantial percentage of the population, including hospitalized patients, residents in long-term care institutions, and those with various neurological or genitourinary disorders. The problems commonly associated with indwelling devices have led us to refer to them as a "one-point restraint." ${ }^{10}$ If there is a need to monitor urine output or to overcome obstructive uropathy, an indwelling (urethral or suprapubic) device is required. Otherwise, there are several other available options, including a condom catheter for male patients. Although indwelling catheters generally cause discomfort, condom catheters tend to fall off, and both are associated with bacteriuria. Surprisingly, no previous randomized, controlled trial that compared the two devices was found. The recent development of a condom catheter that is less apt to dislodge made this an opportune time to undertake such a prospective evaluation.

The primary finding from this trial was that using a condom catheter rather than an indwelling catheter in male inpatients lowered the incidence of bacteriuria, symptomatic UTI, or death. This protective effect was seen primarily in men who did not have dementia. As the first randomized, controlled trial comparing indwelling catheters with condom catheters, this study has important patient safety implications. UTI is the most common hospitalacquired infection in the United States. ${ }^{11,13-15}$ Thus, any intervention that can potentially decrease the incidence of this complication, even in a subgroup of patients, is of obvious benefit. An important secondary finding was that patients reported that an external urinary collection device was more comfortable and less painful than an indwelling catheter. This finding supports previous observations in male patients. ${ }^{9}$

Few previous studies have examined the relative merits of external versus indwelling urinary catheters. In a prospective study published more than 25 years ago, the rate of bacteriuria in men wearing a condom catheter was found to be approximately $12 \%$ per month, but the incidence was much higher in the subgroup of men who frequently manipulated their catheters. ${ }^{25}$ In two parallel noninterventional cohort nursing home studies, the incidence of symptomatic UTI was about two and one-half times greater in men with a chronic indwelling catheter than in men using a condom catheter. ${ }^{19,20}$ Similarly, a more recent prospective but nonrandomized observational study of male patients with a remote spinal cord injury found that those using an indwelling catheter had an almost eight times greater risk of UTI as those using a condom catheter. ${ }^{22} \mathrm{Al}-$ ternatively, a descriptive point-prevalence cross-sectional survey in Danish nursing homes found that the prevalence of UTI was more than two times as high in men wearing a 
Table 4. Patient Satisfaction with the Urinary Collection Device Worn, Based on Responses to a Questionnaire

\begin{tabular}{lrrr}
\hline & \multicolumn{2}{c}{ Yes } \\
\cline { 2 - 3 } & \multicolumn{2}{c}{$\begin{array}{l}\text { Condom Indwelling } \\
\text { Catheter }\end{array}$} & \\
\cline { 2 - 3 } \multicolumn{1}{c}{ Is Your Urinary Catheter: } & \multicolumn{2}{c}{$\%$} & \\
\hline Comfortable? & \multicolumn{2}{c}{ P-value* } \\
Painful? & 59.5 & 57.6 & .02 \\
Convenient? & 78.9 & 36.4 & .02 \\
Restricting your daily activity? & 20.0 & 38.3 & .74 \\
Causing you embarrassment? & 5.3 & 21.9 & .16 \\
& & &
\end{tabular}

${ }^{*}$ Entries are Pearson chi-square or two-sided Fisher exact test (for expected cell counts $<5$ ).

condom catheter as in those with an indwelling catheter. ${ }^{21}$ Unlike randomized trials, point-prevalence studies suffer from several potential biases. Among these is reverse causality: patients with recurrent UTI related to an indwelling catheter may be more likely to be offered another urinary collection method. Second, survival bias may occur if adverse outcomes are indeed associated with using an indwelling catheter. Third, prevalence studies do not take into account time of the exposure (days of catheter use) or time to outcome, both of which are integral to the pathogenesis of this infectious disease.

This study has several potentially important limitations. First, it was only possible to enroll a relatively small number of subjects despite 4 years of intensive recruiting. Among the difficulties encountered were that many potential subjects would not agree to be randomized to one of the types of catheter, usually the indwelling. Some understandably resisted potentially being switched from a catheter that was functional for them. Many patients were ineligible, because they already had bacteriuria at the time of screening or were receiving systemic antimicrobial therapy. To improve enrollment, the research nurse tracked patients with bacteriuria who were receiving antimicrobial therapy and, when the therapy was discontinued, attempted to enroll them, but they were frequently discharged before they could be enrolled. Additionally, a large number of potentially eligible patients were so cognitively impaired that it seemed unlikely that they could provide informed consent. For most of these patients, a surrogate decision maker could not be located in a timely manner or the surrogate would not agree to have the patient participate in the study. Finally, other patients met one of the remaining seven exclusion criteria for enrollment listed in the methods section.

Second, this study was conducted at a single site, an academically affiliated Department of Veterans Affairs hospital; this may limit its generalizability, even to other male populations. Third, a specific type of condom catheter that comes in five sizes and reliably stays in place was studied. Other types of condom catheters may not provide the same benefits. Fourth, this study is only relevant to men, because few clinicians use the external collection devices developed for women. ${ }^{30}$ Fifth, the patients, the providers, and the investigators could not be easily blinded to the type of cath- eter used. Finally, for unclear reasons, randomization resulted in an unequal distribution of dementia in the two patient groups at baseline. However, the data analyses allowed for effect modification by dementia status. Although acknowledging these limitations, most are inherent to studying this clinical question. This experience makes clear why this question has not been addressed adequately in the past, and another randomized trial of this type is unlikely to be undertaken soon.

These findings have potentially important policy implications. For men without dementia, use of a condom catheter rather than an indwelling urinary catheter appears to be highly beneficial in reducing clinically important adverse outcomes, but there may be no benefit in using a condom catheter in patients with dementia. Given the higher level of patient satisfaction with the condom catheter, it should be the urinary collection device of choice for appropriately selected patients. Using a condom catheter in a cooperative man who requires a urinary drainage device is an important part of a comprehensive strategy to reduce the incidence of catheter-related bacteriuria. ${ }^{31,32}$

In conclusion, this randomized trial found that the use of a condom catheter instead of an indwelling catheter in male inpatients was associated with a lower risk of bacteriuria, symptomatic UTI, or death. Although awaiting confirmatory trials, these findings should encourage clinicians to select a condom rather than an indwelling catheter where possible for the millions of men requiring a urinary collection device.

\section{ACKNOWLEDGMENTS}

Financial Disclosure: This project was supported by the Robert Wood Johnson Clinical Scholars Program, the Department of Veterans Affairs, the Mentor Corporation, and the Department of Veterans Affairs/University of Michigan Patient Safety Enhancement Program. Dr. Saint is supported by an Advanced Career Development Award from the Health Services Research and Development Program of the Department of Veterans Affairs.

Author Contributions: Sanjay Saint and Benjamin A. Lipsky: study concept and design, acquisition of subjects, analysis and interpretation of data, and preparation of manuscript. Samuel R. Kaufman: acquisition of data, analysis and interpretation of data, and preparation of manuscript. Mary A. M. Rogers: analysis and interpretation of data and preparation of manuscript. Paul D. Baker and Kathleen Ossenkop: study concept and design and acquisition of subjects.

Sponsor's Role: The funding sources did not control or influence the study design, the data analysis, data interpretation, or any part of this paper. The funding sources had no role in the decision to submit the manuscript for publication.

\section{REFERENCES}

1. Warren JW. Catheter-associated urinary tract infections. Infect Dis Clin North Am 1997;11:609-622.

2. Saint S, Wiese J, Amory JK et al. Are physicians aware of which of their patients have indwelling urinary catheters? Am J Med 2000;109:476-480.

3. Haley RW, Hooton TM, Culver DH et al. Nosocomial infections in U.S. hospitals, 1975-1976: Estimated frequency by selected characteristics of patients. Am J Med 1981;70:947-959. 
4. Warren JW, Steinberg L, Hebel JR et al. The prevalence of urethral catheterization in Maryland nursing homes. Arch Intern Med 1989;149: 1535-1537.

5. Kunin CM, Douthitt S, Dancing J et al. The association between the use of urinary catheters and morbidity and mortality among elderly patients in nursing homes. Am J Epidemiol 1992;135:291-301.

6. Brandeis GH, Baumann MM, Hossain M et al. The prevalence of potentially remediable urinary incontinence in frail older people: A study using the Minimum Data Set. J Am Geriatr Soc 1997;45:179-184.

7. Gammack JK. Use and management of chronic urinary catheters in longterm care: Much controversy, little consensus. J Am Med Dir Assoc 2003;4 (2 Suppl):S52-S59.

8. Johnson TM, Ouslander JG, Uman GC et al. Urinary incontinence treatment preferences in long-term care. J Am Geriatr Soc 2001;49:710-718.

9. Saint S, Lipsky BA, Baker PD et al. Urinary catheters: What type do men and their nurses prefer? J Am Geriatr Soc 1999;47:1453-1457.

10. Saint S, Lipsky BA, Goold SD. Indwelling urinary catheters: A one-point restraint? Ann Intern Med 2002;137:125-127.

11. Saint S, Chenoweth CE. Biofilms and catheter-associated urinary tract infections. Infect Dis Clin North Am 2003;17:411-432.

12. Krieger JN, Kaiser DL, Wenzel RP. Urinary tract etiology of bloodstream infections in hospitalized patients. J Infect Dis 1983;148:57-62.

13. Burke JP. Patient safety: Infection control - a problem for patient safety. N Engl J Med 2003;348:651-656.

14. Haley RW, Culver DH, White JW et al. The nationwide nosocomial infection rate. A new need for vital statistics. Am J Epidemiol 1985;121: 159-167.

15. Gerberding JL. Hospital-onset infections: A patient safety issue. Ann Intern Med 2002;137:665-670.

16. Warren JW. Catheter-associated bacteriuria in long-term care facilities. Infect Control Hosp Epidemiol 1994;15:557-562.

17. Saint S. Clinical and economic consequences of nosocomial catheter-related bacteriuria. Am J Infect Control 2000;28:68-75.

18. Tambyah PA, Knasinski V, Maki DG. The direct costs of nosocomial catheterassociated urinary tract infection in the era of managed care. Infect Control Hosp Epidemiol 2002;23:27-31
19. Ouslander JG, Greengold B, Chen S. Complications of chronic indwelling urinary catheters among male nursing home patients: A prospective study. J Urol 1987;138:1191-1195.

20. Ouslander JG, Greengold B, Chen S. External catheter use and urinary tract infections among incontinent male nursing home patients. J Am Geriatr Soc 1987;35:1063-1070.

21. Zimakoff J, Stickler DJ, Pontoppidan B et al. Bladder management and urinary tract infections in Danish hospitals, nursing homes, and home care: A national prevalence study. Infect Control Hosp Epidemiol 1996;17:215-221.

22. Esclarin De Ruz A, Garcia Leoni E, Herruzo Cabrera R. Epidemiology and risk factors for urinary tract infection in patients with spinal cord injury. J Urol 2000;164:1285-1289.

23. Jamison J, Maguire S, McCann J. Catheter policies for management of long term voiding problems in adults with neurogenic bladder disorders. Cochrane Database Syst Rev 2004;(2): CD004375.

24. Warren JW. Urethral catheters, condom catheters, and nosocomial urinary tract infections. Infect Control Hosp Epidemiol 1996;17:212-214.

25. Hirsh DD, Fainstein V, Musher DM. Do condom catheter collecting system cause urinary tract infection? JAMA 1979;242:340-341.

26. Lipsky BA, Ireton RC, Fihn SD et al. Diagnosis of bacteriuria in men: Specimen collection and culture interpretation. J Infect Dis 1987;155:847-854.

27. Stark RP, Maki DG. Bacteriuria in the catheterized patient. What quantitative level of bacteriuria is relevant? N Engl J Med 1984;311:560-564.

28. Nicolle LE, Harding GK, Kennedy J et al. Urine specimen collection with external devices for diagnosis of bacteriuria in elderly incontinent men. J Clin Microbiol 1988;26:1115-1119.

29. Ouslander JG, Greengold BA, Silverblatt FJ et al. An accurate method to obtain urine for culture in men with external catheters. Arch Intern Med 1987;147:286-288.

30. Johnson DE, Muncie HL, O'Reilly JL et al. An external urine collection device for incontinent women. Evaluation of long-term use. J Am Geriatr Soc 1990; 38:1016-1022.

31. Warren JW. Catheter-associated urinary tract infections. Int J Antimicrob Agents 2001;17:299-303.

32. Saint S, Lipsky BA. Preventing catheter-related bacteriuria: Can we? Should we? How? Arch Intern Med 1999;159:800-808. 